\title{
Fibre laser focus
}

\author{
With their ultrafast and high-power characteristics, fibre lasers are penetrating conventional laser \\ markets as well as opening up exciting new opportunities.
}

This month's Nature Photonics is a focus issue dedicated to the field of fibre lasers. Over the past several years, we have seen fibre laser concepts (for example, supercontinuum sources) mature to commercialization, and there is no shortage of new ideas and applications for fibre lasers.

Nature Photonics spoke to Anatoly Grudinin, founder of the fibre laser company Fianium, to gain some insight into the transition from academic research on fibre lasers to commercialization. Grudinin emphasizes that despite the photonics market experiencing some vicissitudes in the past decade, fibre lasers are going strongly. In fact, Grudinin says that Fianium was hardly affected by the 2008 global financial crisis and that there were some advantages to starting an optics company in the wake of the telecom bubble burst in the early 2000s. Grudinin identifies new industrial applications, including biomedical applications (for example, surgery) and industrial materials processing, as being existing or expected growth areas. In addition, the company has delivered over 1,000 supercontinuum sources. Fibre lasers have some obvious advantages, such as their simplicity, low cost and high reliability, but some challenges lie ahead. Although nonlinearity can generate a lot of rich physics and novel concepts (for example, supercontinuum sources), Grudinin notes that it is the main obstacle to developing many new fibre laser ideas. The interview can be found on page 846 of this issue.

One of the reasons for the increasing popularity of fibre lasers for industrial and medical applications, as well as pure scientific research, is their ability to generate ultrafast pulses. On page 868 of this issue, Martin Fermann and Ingmar Hartl review the essential components and mechanisms of ultrafast fibre laser systems. They highlight the importance of largemode-area fibres, especially in chirpedpulse-amplification schemes. In addition to describing the physics, they explore the commercial applications of industrial micromachining and medical imaging. They also discuss emerging applications in the mid-infrared and extreme-ultraviolet spectral regions, which are facilitated by frequency shifting of fibre frequency combs.

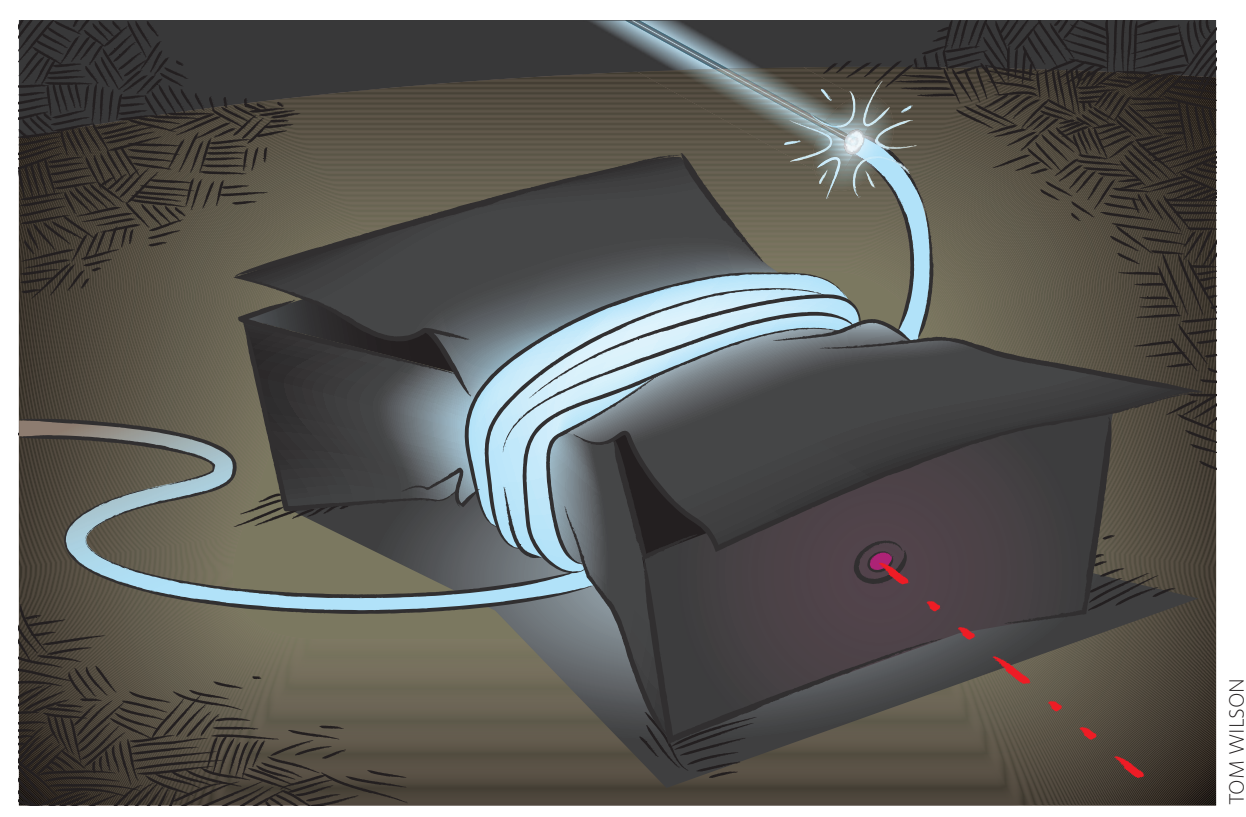

The ultrafast characteristic of pulsed lasers is obviously interesting, but some researchers and users require extremely high average powers. In fact, fibre lasers are known for their ability to deliver very high average powers with good beam quality, as Cesar Jauregui, Jens Limpert and Andreas Tünnermann explain in their Review on page 861 . High power is particularly important for industrial and defence applications, but it is also applicable for scientific applications. The authors note that the success of fibre lasers is the result of both the inherent useful characteristics of fibres as an active medium and of many groups striving to overcome the limitations of fibres. Their Review discusses these limitations and recounts the history that led to fibre lasers being able "to generate the highest diffraction-limited average power achieved to date by solid-state lasers."

Nonlinear microscopy, a major area of photonics, has seen significant development in recent years. Recently, fibre lasers have proved particularly effective sources for this application. On page 875 , Chris $\mathrm{Xu}$ and Frank Wise review the advances in fibre laser properties that are particularly relevant for nonlinear microscopy and that have resulted in advances in bioimaging. They explain that fibre lasers have characteristics that are particularly suitable for biological imaging, and that systems based on fibre lasers are robust, inexpensive and easily integrated.

We shouldn't forget that fibre lasers and their interesting characteristics have been realized through a lot of developments that have involved many aspects besides just fibre issues. In their Commentary on page 842, Amos Martinez and Zhipei Sun cover the development of saturable absorbers for achieving mode locking in fibre lasers. They emphasize that carbon nanotubes and graphene are good emerging options for saturable absorbers in ultrafast fibre lasers. These materials have unique electrical and optical properties, which have recently been discussed a lot in various contexts; their properties are expected to enhance the performance of saturable absorbers. Other key factors of these saturable absorbers are their simplicity and flexibility of fabrication and ease of integration into fibre lasers.

Grudinin mentions that fibre lasers have very few limitations and that there is great potential for further improvements in their performance. It will be interesting to observe the extent to which fibre lasers penetrate domains that conventional lasers presently dominate. We also look forward to discovering what entirely new directions will be spawned by ongoing scientific progress. 